\title{
New insights on potential permanent pacemaker predictors in TAVR using the largest self-expandable device
}

\author{
Verena Veulemans $^{1 \#}$, Derk Frank ${ }^{2,3 \#}$, Hatim Seoudy ${ }^{2,3}$, Steffen Wundram $^{2}$, Kerstin Piayda ${ }^{1}$, Oliver Maier ${ }^{1}$, \\ Christian Jung ${ }^{1}$, Amin Polzin ${ }^{1}$, Norbert Frey ${ }^{4,5}$, Malte Kelm ${ }^{1,6}$, Tobias Zeus ${ }^{1}$ \\ ${ }^{1}$ Division of Cardiology, Pulmonology and Vascular Medicine, Heinrich Heine University, Medical Faculty, Düsseldorf, Germany; ${ }^{2}$ Department \\ of Internal Medicine III, Cardiology and Angiology, University Hospital Schleswig-Holstein, Kiel, Germany; ${ }^{3}$ DZHK (German Centre for \\ Cardiovascular Research), Partner Site Hamburg/Kiel/Lübeck, Kiel, Germany; ${ }^{4}$ Department of Cardiology, Angiology and Pneumology, Internal \\ Medicine III, Medical Hospital, Heidelberg University Hospital, Heidelberg, Germany; ${ }^{5}$ DZHK (German Centre for Cardiovascular Research), \\ Partner Site Heidelberg/Mannheim, Heidelberg, Germany; ${ }^{6}$ CARID (Cardiovascular Research Institute Düsseldorf), Düsseldorf, Germany \\ Contributions: (I) Conception and design: V Veulemans, D Frank, H Seoudy; (II) Administrative support: C Jung, T Zeus; (III) Provision of study \\ materials or patients: V Veulemans, D Frank; (IV) Collection and assembly of data: V Veulemans, H Seoudy, S Wundram; (V) Data analysis and \\ interpretation: V Veulemans, D Frank, H Seoudy; (VI) Manuscript writing: All authors; (VII) Final approval of manuscript: All authors. \\ \#These authors contributed equally to this work. \\ Correspondence to: Tobias Zeus, MD. Division of Cardiology, Pulmonology and Vascular Medicine, Heinrich Heine University, Medical Faculty, \\ Moorenstr. 5, Düsseldorf 40225, Germany. Email: Zeus@med.uni-duesseldorf.de.
}

Background: Post-procedural conduction disorders following transcatheter aortic valve replacement (TAVR) still remain frequent, especially using the largest self-expandable device (Medtronic Corevalve Evolut $\mathrm{R}^{\mathrm{TM}}, 34 \mathrm{~mm}$, STHV-34). We, therefore, assessed previously described, predictive factors of permanent pacemaker (PPM) implantation in the context of the STHV-34, including calcification distribution, implantation depth and membranous septum length (MSL).

Methods: We performed a dual centre analysis of 130 of 182 consecutive patients treated with STHV-34, further stratified into subjects without post-procedural PPM (-PPM n=100, 76.9\%) and those requiring postprocedural PPM (+PPM n=30, 23.1\%). These events were further analyzed by univariate and multivariate analysis according to several underlying conditions.

Results: Multivariate analysis only depicted previous right bundle branch block [RBBB; OR: 11.52 (2.6350.44), $\mathrm{P}=0.001$ ] and eccentricity index of the left ventricular outflow tract (LVOT-EI) >0.3 [OR: 3.07 (1.227.77), $\mathrm{P}=0.018$ ] as highly predictive for $\mathrm{PPM}$-need, being also confirmed by c-statistics [area under the curve $(\mathrm{AUC})=0.68 ; 95 \%$ confidence interval $(\mathrm{CI}): 0.57-0.80 ; \mathrm{P}=0.0025]$. There was only moderate correlation of implantation depth over the MSL in terms of PPM prediction $(r=0.23 ; \mathrm{P}<0.0001)$.

Conclusions: This study offers new insights into potential PPM predictors using the STHV-34: previous RBBB and a pronounced LVOT-EI were independent predictors of PPM, while most of the previously reported determinants failed to predict PPM-need including MSL and implantation depth.

Keywords: Atrio-ventricular block; imaging modalities; transcatheter aortic valve implantation (TAVI); transcatheter aortic valve replacement (TAVR); elderly

Submitted Jul 31, 2020. Accepted for publication Oct 25, 2020.

doi: $10.21037 / \mathrm{cdt}-20-680$

View this article at: http://dx.doi.org/10.21037/cdt-20-680

\section{Introduction}

Aortic valve stenosis (AS) is the most common valvular heart disease in western countries with increasing prevalence (1).
Transcatheter aortic valve replacement (TAVR) is well known to be the treatment option of choice in inoperable and high-risk patients and currently shows favorable 
outcomes in intermediate- or even low-risk cohorts $(2,3)$. Technological advancements such as smaller sheath sizes, retrievable valves, and new skirt techniques promise outcome optimization (4), also including coverage of large annulus sizes. The recently released self-expandable $34 \mathrm{~mm}$ Evolut $^{\mathrm{TM}} \mathrm{R}$ valve (Medtronic, Minneapolis, MN, USA; STHV-34) extended the annulus diameter range up to $30 \mathrm{~mm}$. The STHV-34 is characterized by high procedural success, excellent hemodynamic performance with low flow velocities and low early complication rates (5-7). However, postprocedural conduction disorders following TAVR with self-expandable devices remain frequent, in the range of $25-35 \%$ using old-generation $(8,9)$ and $15-35 \%$ using newgeneration devices, respectively $(5-7,10,11)$. Determinants of new permanent pacemaker (PPM) implantation after TAVR with the STHV-34 might be linked to certain technical, anatomical and procedural conditions. The recently described patient-specific MIDAS (MInimizing Depth According to the membranous Septum) approach reported very low and predictable rates of PPM compared to previously published data on self-expanding TAVR prostheses (12). However, the impact of MIDAS on PPM probability using the STHV34 is unknown. We, therefore, aimed to evaluate previously described, well-established predictive factors and examined other influencing parameters of PPM in the context of STHV-34. We present the following article in accordance with the MDAR reporting checklist (available at http:// dx.doi.org/10.21037/cdt-20-680).

\section{Methods}

\section{Study population}

We performed a dual centre analysis (Düsseldorf: $n=126$; Kiel: $\mathrm{n}=56)$ in 182 consecutive patients treated with the STHV-34 between March 2017 and September 2019. Patients with pre-existing PPM ( $\mathrm{n}=36 ; 19.8 \%)$ were excluded to ensure a true analysis of PPM determinants. Furthermore, patients with a congenital bicuspid aortic valve $(\mathrm{n}=10 ; 5.5 \%)$ and non-suitable multi-slice CT (MSCT)-quality ( $\mathrm{n}=2$; $1.1 \%)$ were also not accepted for our analysis. For reasons of comparability and to avoid bias concerning (relative) pre-existing pacemaker or ICD/CRT indication regarding severely reduced left ventricular ejection fraction, patients who received post-implantation PPM due to hemodynamic relevant bradyarrhythmia and other disturbances were also excluded $(\mathrm{n}=4 ; 2.2 \%)$. The final study population consisted of 130 patients without previous PPM (79.2\%) who underwent TAVR with the STHV-34. Patients were further stratified into subjects without post-procedural PPM $(-\mathrm{PPM} \mathrm{n}=100,76.9 \%)$ and those requiring post-procedural PPM (+PPM n=30, 23.1\%). All procedures were performed according to current guidelines and under local anesthesia.

All patients provided written informed consent for TAVR and the use of clinical, procedural, and follow-up data for research. The study procedures were in accordance with the Declaration of Helsinki (as revised in 2013). The institutional Ethics Committees of the HeinrichHeine University (Düsseldorf, 4080) as well as ChristianAlbrechts-University (Kiel) approved the study protocol.

The study endpoint was defined as determinants for PPM implantation in STHV-34 patients.

\section{MSCT image acquisition protocol}

Preprocedural cardiac CT was routinely performed as native and contrast-enhanced MSCT images in all patients before TAVR. CT data were obtained according to TAVR-related standardized recommendations for MSCT image acquisition (13). Either a 128-slice, single source CT-scanner with a temporal resolution of $150 \mathrm{~ms}$ and a collimation of $128 \mathrm{~mm} \times 0.6 \mathrm{~mm}$ ("SOMATOM Definition AS+", Siemens Healthcare, Forchheim, Germany) or a 256-slice, dual source CT-scanner with a temporal resolution of $75 \mathrm{~ms}$ and a collimation of $2 \mathrm{~mm} \times 128 \mathrm{~mm}$ $\times 0.6 \mathrm{~mm}$ ("SOMATOM Definition Flash", Siemens Healthcare, Forchheim, Germany) were used.

\section{Procedural details and 3D image analysis of MSCT}

Resheathing was defined as retrieval of only a part of the delivered valve into the capsule. Recapture was defined as complete retrieval of the valve with the intention to recross the aortic valve. Pre- and post-dilatation processes were assessed and the final depth of device implantation (ID) was calculated in projection towards the native aortic annulus leaflets [noncoronary cusp (NCC); left coronary cusp (LCC)] by final aortogram in a perpendicular view on the prosthesis and the native anatomy.

MSCT images were transferred to a dedicated workstation for evaluation (3mensio Structural Heart ${ }^{\mathrm{TM}}$, Pie Medical Imaging BV, Maastricht, The Netherlands) and reconstructed in the coronal, sagittal and axial plane. Reconstruction was gained from diastolic phase. Aortic root dimensions and the aortic root angulation (14) were determined. Eccentricity indexes (EIs) were calculated using 

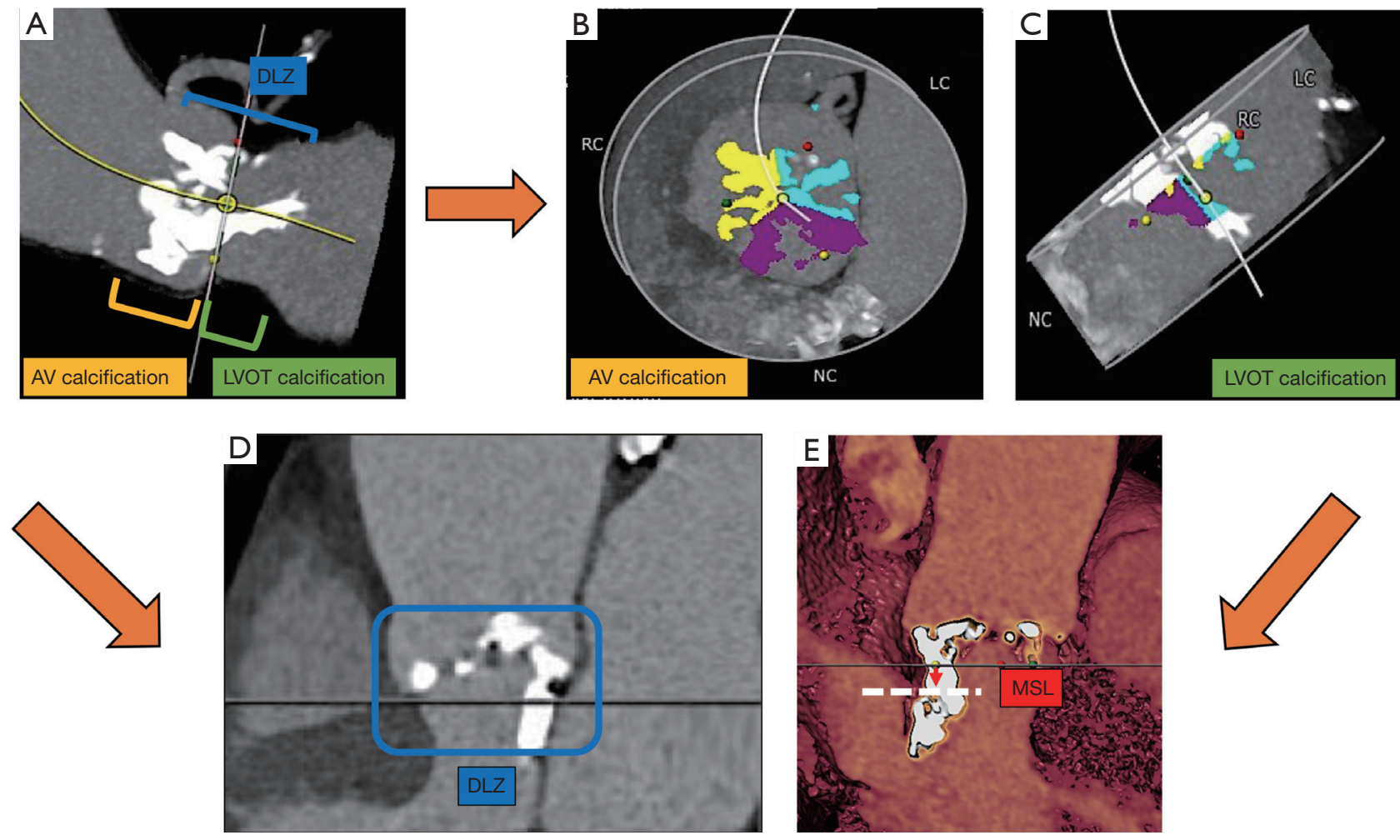

Figure 1 Visualization of aortic valve calcium and DLZ quantification. (A) Calcium amount of the aortic valve (yellow bracket) and the upper LVOT (green bracket) was assessed recalculating AU for overall—and separated leaflet calcium burden (NCC, LCC, RCC) using the "Mercedes Benz" tool for localisation (B). (D) Calcifications of the DLZ (blue bracket) were calculated as the leaflet and upper LVOT region (C). The MSL (red bracket) was measured by determining the thinnest part of the interventricular septum on the perpendicular annular plane (E). DLZ, device landing zone; LVOT, left ventricular outflow tract; AU, Agatston Units; NCC, noncoronary cusp; LCC, left coronary cusp; RCC, right coronary cusp; MSL, membranous septum length; AV, aortic valve.

the shortest and longest diameters (short/long axis) of the annulus and left ventricular outflow tract (LVOT). Valve oversizing was calculated in percent as '(prosthesis size native annulus size/native annulus size) $\times 100 \%$ '.

We assessed the calcium amount of the aortic valve and the upper LVOT within $1 \mathrm{~cm}$ under annulus, recalculating Agatston Units (AU) adapted from the calcium volume on given Hounsfield Units (HU) for overall, and separated leaflet calcium burden using the "Mercedes Benz" tool for localization. A pre-specific threshold of at least 600 HU was set to account for the hyperdensity of the applied contrast medium as practicable approach according to current research data (15). All MSCT-reconstructions and depending analyses were done by experienced level 3 readers, for the most part prospectively, partly retrospective in case of missing data. The device landing zone (DLZ) calcification was calculated as previously described (16) and also separated into segments of calcification site. The membranous septum length (MSL) was measured by determining the thinnest part of the interventricular septum on the perpendicular annular plane according to the MIDAS-approach as previously described (12). Please see also the illustrated Figure 1.

\section{Management of patient care and allocation to PPI}

In general, patients receive 24-72 hours monitoring depending on their risk profile, remaining at least 12 hours on the immediate care unit. In high risk profiles (higher-grade calcification burden, large device, pre-existing conduction disturbances) and persisting pacemaker dependency and/or low-output syndrome, PPM was conducted. 


\section{Statistical analysis}

Continuous data were described by mean and standard deviation, median or upper and lower $95 \%$ confidence interval (CI) (interquartile ranges) and categorical variables by frequencies and percentages. Continuous variables were compared using a Student's $t$-test or Kolmogorov-Smirnov test depending on variable distribution in a heterogenous sample size. Categorical variables were compared using Fishers' exact test. For correlations of interest, Spearman correlation coefficients were calculated. Correlation coefficients of 0.8 to 1.0 and 0.5 to 0.8 , respectively, indicate a very strong and strong positive correlation between two variables, whereas coefficients between 0.2 to 0.5 and 0.0 to 0.2 suggest medium and small correlations, respectively. The influence on PPM was tested by univariate and binary multivariate logistic regression analysis. Covariates associated with PPM in the univariate analysis $(\mathrm{P}<0.1)$ and highly suspected covariates were entered into the multivariate model. Receiver operating characteristic (ROC) curves were described as c-indices [area under the curve (AUC)] with 95\% CIs. The data analysis was performed using the statistical software SPSS (version 23.0, SPSS Inc., Chicago, IL, USA) and GraphPad Prism (version 7.0, Graphpad Software, San Diego, CA, USA). All statistical tests were two-tailed, and a value of $\mathrm{P}<0.05$ was considered statistically significant.

\section{Results}

\section{Baseline characteristics—clinical, functional and rhythm data}

Patients' baseline characteristics did not differ except of previous left (+PPM/-PPM: LBBB $0.0 \%$ vs. $15.0 \%$; $\mathrm{P}=0.022)$ or right bundle branch block $(+\mathrm{PPM} /-\mathrm{PPM}$ : RBBB $26.7 \%$ vs. $3.0 \%$; $\mathrm{P}<0.0001)$. First- and seconddegree atrioventricular block (AVB) type I did not differ significantly between both groups. Furthermore, no centre-effect was apparent. A full overview of baseline characteristics is displayed in Table 1.

\section{Baseline characteristics—procedural and MSCT data}

Patients who underwent new PPM implantation showed no difference concerning implantation depth, MSL, frequency of pre- or post-dilatation, and resheath/recapture maneuvers as compared to -PPM patients by univariate analysis. There was also no association between new PPM and the degree of oversizing or association regarding bioprosthesis to LVOT diameter, EIs, or aortic root entrance angle. Again, there was no relevant centre-effect $(\mathrm{P}=0.382$; OR: $1.48 ; 95 \%$ CI: 0.66-3.36) estimated by univariate analysis. Therefore, the centre-effect was not integrated into the multivariate analysis with a $\mathrm{P}>0.1$.

The correlation matrix of need for PPM implantation related to prosthesis implantation depth towards NCC (Spearman Rho: $\mathrm{r}=-0.02 ; \mathrm{P}=0.517$ ), MSL (Spearman Rho: $\mathrm{r}=-0.46 ; \mathrm{P}<0.0001$ ), MSL to ID distance (Spearman Rho: $\mathrm{r}=0.03 ; \mathrm{P}=0.222$ ), and ID $\geq$ MSL (Spearman Rho: $\mathrm{r}=0.25$; $\mathrm{P}<0.0001$ ) as well as a calcified ID $\geq \mathrm{MSL}_{\mathrm{Ca}}$ (Spearman Rho: $\mathrm{r}=0.04 ; \mathrm{P}=0.117$ ) are further visualized in Figure $2 \mathrm{~A}$.

Linear parameters were further categorized into lower and upper median thresholds. Univariate analysis depicted the existence of baseline RBBB, LVOT-EI $>0.30$, LCC calcium burden $<762 \mathrm{AU}$, and DLZ-LCC $<797 \mathrm{AU}$ as possible influencers on PPM probability.

Multivariate analysis only depicted previous RBBB [OR: 11.52 (2.63-50.44); $\mathrm{P}=0.001]$ and LVOT-EI $>0.3$ [OR: 3.07 $(1.22-7.77) ; \mathrm{P}=0.018]$ as highly predictive for $\mathrm{PPM}$ need. For further details please see also Table 2. C-statistic of the combined independent predictors confirmed the association with need for PPM implantation following TAVR (Figure 2B: $\mathrm{AUC}=0.68 ; 95 \% \mathrm{CI}: 0.57-0.80 ; \mathrm{P}=0.0025)$, further strengthened when taken a LCC calcium burden $<762 \mathrm{AU}$ from the univariate analysis into account (Figure $2 C$ : AUC $=0.72 ; 95 \%$ CI: $0.62-0.82 ; \mathrm{P}=0.0002)$.

\section{Post-procedural characteristics of patients with need for PPM}

All patients of the final study-cohort had their PPM implanted during the same index hospitalization with an average of 2.23 days following TAVR. All indications for PPM implantation are illustrated in Table 3. Nearly all patients with preexistent single RBBB ( $\mathrm{n}=2,6.7 \%)$, RBBB in combination with atrial fibrillation (AF) $(\mathrm{n}=5,16.7 \%)$, or AVB I-II.I ${ }^{\circ}(\mathrm{n}=1,3.3 \%)$ suffered immediate intra- or post-procedural third degree AVB $(n=8 ; 26.7 \%)$ without functional recovery. Interestingly, a high number of patients with normal sinus rhythm (SR) developed also postprocedural AVB II-III ${ }^{\circ}$ with need for PPM ( $\left.\mathrm{n}=12 ; 40.0 \%\right)$.

\section{Sub-analysis of patients with need for PPM and previous SR}

The correlation matrix is illustrated in Figure S1A, still offering no associations of implantation depth and MSL in terms of PPM dependency. In 12 patients with previous SR, 
Table 1 Clinical, functional and intra- and periprocedural characteristics

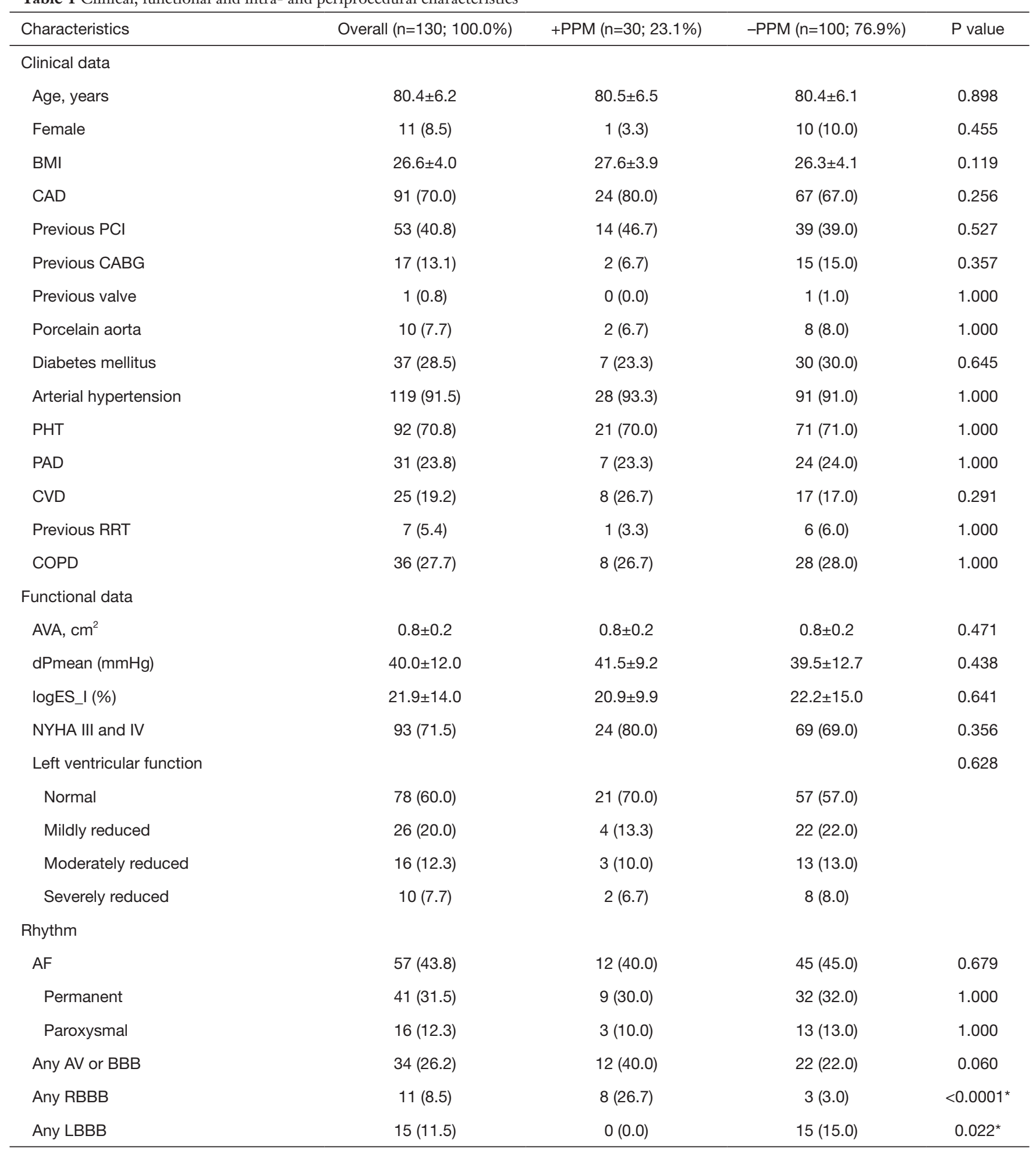

Table 1 (continued) 
Table 1 (continued)

\begin{tabular}{|c|c|c|c|c|}
\hline Characteristics & Overall $(n=130 ; 100.0 \%)$ & +PPM (n=30; 23.1\%) & -PPM (n=100; 76.9\%) & $P$ value \\
\hline Pre-dilatation & $67(51.5)$ & $18(60.0)$ & $48(48.0)$ & 0.124 \\
\hline Post-dilatation & $27(20.8)$ & $8(26.7)$ & $19(19.0)$ & 0.442 \\
\hline Resheath/recapture & $25(19.2)$ & $4(13.3)$ & $21(21.0)$ & 0.436 \\
\hline Implant depth-LCC (mm) & $6.9 \pm 3.1$ & $7.0 \pm 3.7$ & $6.9 \pm 2.9$ & 0.890 \\
\hline MSL (mm) & $6.6 \pm 2.6$ & $6.7 \pm 2.7$ & $6.7 \pm 2.5$ & 0.853 \\
\hline MSL-ID (mm) & $0.0[-1.0$ to 0.0$]$ & $0.0[0.0$ to 1.0$]$ & $0.0[-1.0$ to 0.0$]$ & 0.976 \\
\hline$I D \geq M S L$ & $39(30.0)$ & $9(30.0)$ & $30(30.0)$ & 1.000 \\
\hline Valve oversizing by perimeter (\%) & $23.1 \pm 4.9$ & $23.0 \pm 3.8$ & $23.1 \pm 5.1$ & 0.929 \\
\hline THV-d/LVOT-d & $1.3 \pm 0.2$ & $1.3 \pm 0.1$ & $1.3 \pm 0.2$ & 0.983 \\
\hline Annulus El & $0.2 \pm 0.1$ & $0.2 \pm 0.1$ & $0.2 \pm 0.1$ & 0.715 \\
\hline LVOT-EI & $0.3 \pm 0.1$ & $0.3 \pm 0.2$ & $0.3 \pm 0.1$ & 0.938 \\
\hline Annulus/LVOT-EI & $0.8 \pm 0.5$ & $0.7 \pm 0.4$ & $0.9 \pm 0.6$ & 0.124 \\
\hline Aortic root $\left({ }^{\circ}\right)$ & $51.2 \pm 8.1$ & $50.8 \pm 8.0$ & $51.4 \pm 8.2$ & 0.722 \\
\hline Calcification AV total (AU) & $2,565[1,785-3,896]$ & $2,536[1,706-4,087]$ & $2,575[1,813-3,886]$ & 0.806 \\
\hline NCC & $991[583-1,563]$ & $1,024[680-1,586]$ & $958[573-1,567]$ & 0.898 \\
\hline $\mathrm{RCC}$ & $0[0-7]$ & $0[0-14]$ & $0[0-6]$ & 0.256 \\
\hline $\mathrm{DLZ}$ total $(\mathrm{AU})$ & $2,756[1,982-4,063]$ & $2,810[1,865-4,169]$ & $2,756[2,001-4,170]$ & 0.856 \\
\hline NCC & $1,129[673-1,629]$ & $1,091[776-1,586]$ & $1,129[610-1,639]$ & 0.941 \\
\hline LCC & $797[458-1,309]$ & $666[366-1,136]$ & $930[490-1,363]$ & 0.574 \\
\hline $\mathrm{RCC}$ & $772[467-1,201]$ & $778[507-1,188]$ & $772[447-1,251]$ & 0.815 \\
\hline Severe DLZ total & $52(40.0)$ & $13(43.3)$ & $39(39.0)$ & 0.677 \\
\hline
\end{tabular}

Values are mean $\pm \mathrm{SD}$, median (interquartile ranges) or $\mathrm{n}(\%) .{ }^{*}$, significant $\mathrm{P}$ value $<0.05$. PPM, permanent pacemaker; BMI, body mass index; CAD, coronary artery disease; PCI, percutaneous coronary intervention; CABG, coronary artery bypass graft; PHT, pulmonary hypertension; PAD, peripheral artery disease; CVD, cerebrovascular disease; RRT, renal replacement therapy; COPD, chronic obstructive pulmonary disease; AVA, aortic valve area; d-, diameter; AF, atrial fibrillation; AV, aortic valve; BBB, bundle branch block; RBBB, right bundle branch block; LBBB, left bundle branch block; NCC, noncoronary cusp; LCC, left coronary cusp; MSL-ID, difference between membranous septal length and device implantation depth; Ca, calcified; MSCT, multi-slice CT; THV, transcatheter heart valve; LVOT-EI, eccentricity index of the left ventricular outflow tract; AU, Agatston Units; RCC, right coronary cusp; DLZ, device landing zone. 
A

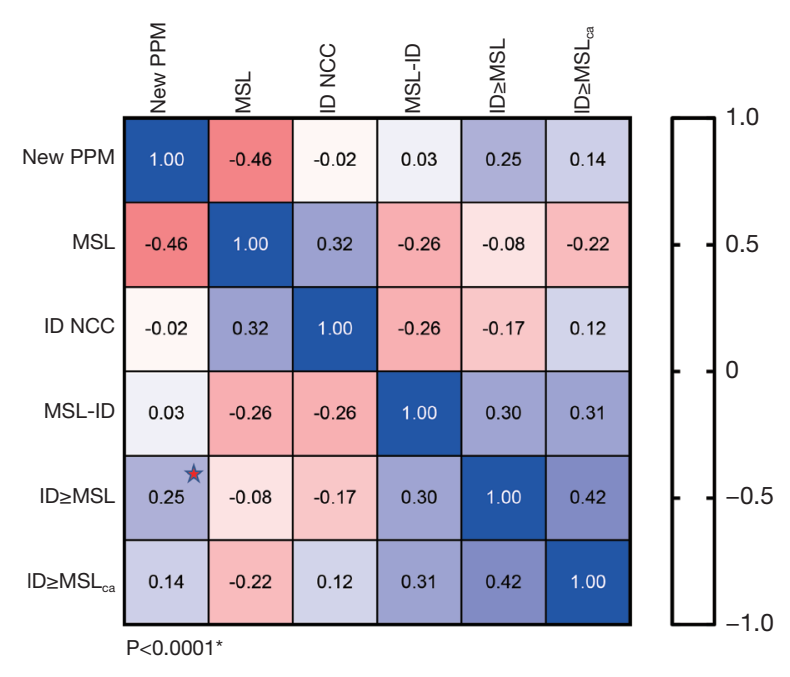

B

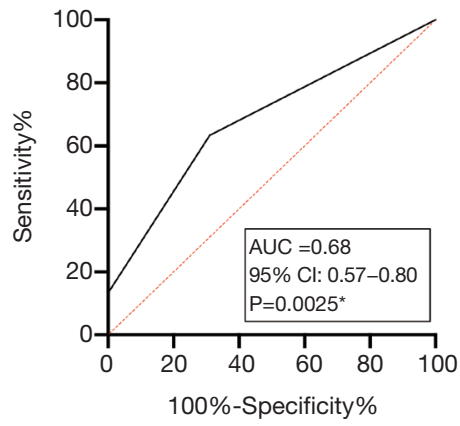

C

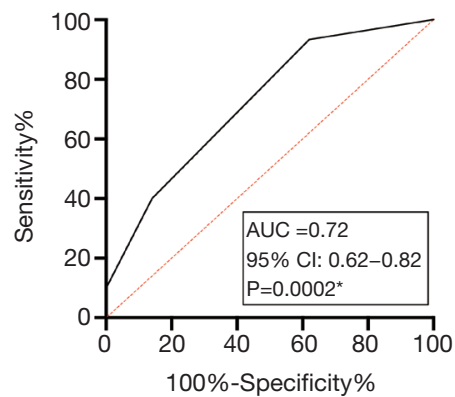

Figure 2 Association of PPM need with potential and determined predictive factors. (A) Correlation matrix of ID towards NCC, MSL, MSL to ID distance, ID $\geq$ MSL, and ID $\geq$ MSLCa. (B) Combined c-statistics of the independent predictors for PPM need (RBBB and LVOT-EI $>0.3$ ). (C) Combined c-statistics of the independent (RBBB and LVOT-EI $>0.3$ ) and a dependent (LCC calcium burden $<762 \mathrm{AU}$ ) predictor for PPM need. *, significant P value $<0.05$. ID, implantation depth; NCC, noncoronary cusp; MSL, membranous septum length; PPM, permanent pacemaker; RBBB, right bundle branch block; LVOT-EI, eccentricity index of the left ventricular outflow tract; LCC, left coronary cusp; AU, Agatston units; AUC, area under the curve; CI, confidence interval.

Table 2 Uni- and multivariate logistic regression analysis of PPM-predictors

\begin{tabular}{|c|c|c|c|c|}
\hline Predictor & \multicolumn{2}{|c|}{ Univariate analysis } & \multicolumn{2}{|c|}{ Multivariate analysis } \\
\hline Any RBBB & $11.76(2.88-47.93)$ & $0.001^{*}$ & $11.52(2.63-50.44)$ & $0.001^{*}$ \\
\hline LVOT-EI >0.3 & $2.57(1.11-5.95)$ & $0.027^{*}$ & 3.07 (1.22-7.77) & $0.018^{*}$ \\
\hline LCC <762 AU & $2.44(1.04-5.75)$ & $0.041^{*}$ & - & - \\
\hline
\end{tabular}

*, significant $\mathrm{P}$ value $<0.05$. PPM, permanent pacemaker; Cl, confidence interval; RBBB, right bundle branch block; LVOT-El, eccentricity index of the left ventricular outflow tract; LCC, left coronary cusp; AU, Agatston Units; DLZ, device landing zone.

univariate analysis depicted pronounced obesity, pre- and post-dilatation processes as well as LCC calcium burden $<762 \mathrm{AU}$, and DLZ-LCC $<757 \mathrm{AU}$ as possible influencers on PPM probability, while only pre-dilatation [OR: 8.53 (1.23-59.41); $\mathrm{P}=0.030]$ and LCC calcium burden $<762 \mathrm{AU}$ [OR: 16.12 (1.74-149.67); $\mathrm{P}=0.014]$ were identified as independent predictors for PPM need. For further details please see also Table S1. C-statistic of the combined independent predictors confirmed this association
(Figure S1B: AUC =0.76; 95\% CI: 0.60-0.93; P=0.0061). Please see also the central illustration Figure 3 giving an overview on the main cohort and the sub-cohort with their identified risk factors.

\section{Discussion}

The present study evaluating predictive factors for postprocedural PPM implantation in the context of the largest 
Table 3 Post-procedural sub-analysis of patients with need for PPM (rhythm entities and distribution)

\begin{tabular}{|c|c|c|c|c|c|c|}
\hline Parameters & Baseline rhythm & \multicolumn{5}{|c|}{ Post-procedural rhythm } \\
\hline Permanent AF & $6(20.0)$ & $1(3.3)$ & $0(0.0)$ & $0(0.0)$ & $5(16.7)$ & - \\
\hline Paroxysmal AF & $1(3.3)$ & $0(0.0)$ & $0(0.0)$ & $0(0.0)$ & $1(3.3)$ & - \\
\hline Perm. AF + RBBB & $3(10.0)$ & $0(0.0)$ & $0(0.0)$ & $0(0.0)$ & $3(10.0)$ & - \\
\hline RBBB & $2(6.7)$ & $0(0.0)$ & $0(0.0)$ & $0(0.0)$ & $2(6.7)$ & - \\
\hline LBBB & $0(0.0)$ & $0(0.0)$ & $0(0.0)$ & $0(0.0)$ & $0(0.0)$ & - \\
\hline AVB I-II. ${ }^{\circ}$ & $3(10.0)$ & $0(0.0)$ & $0(0.0)$ & $0(0.0)$ & $3(10.0)$ & - \\
\hline AVB I-II. $1^{\circ}+$ RBBB & $1(3.3)$ & $0(0.0)$ & $0(0.0)$ & $0(0.0)$ & $1(3.3)$ & - \\
\hline
\end{tabular}

Values are $\mathrm{n}(\%)$. *, significant $\mathrm{P}$ value $<0.05$. PPM, permanent pacemaker; RBBB, right bundle branch block; LBBB, left bundle branch block; AVB, atrioventricular block; AF, atrial fibrillation.
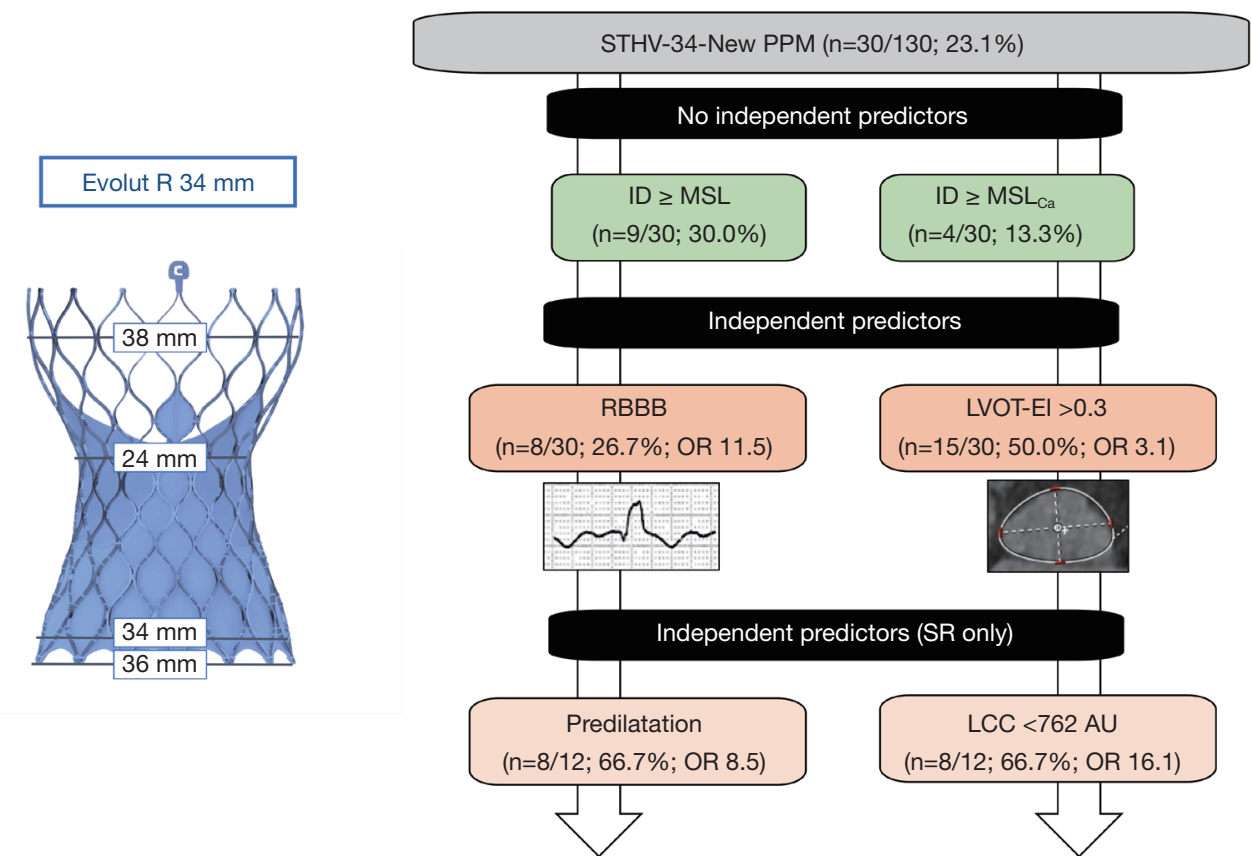

Figure 3 Central illustration. Identified independent risk factors for new PPM in the STHV-34 (stent frame picture as courtesy by Medtronic ${ }^{\circledR}$ ) cohort separated into patients with conduction disturbances and SR. Relation of ID to MSL, even when calcified, was no dependent or independent predictor. PPM, permanent pacemaker; SR, sinus rhythm; ID, implantation depth (related to the NCC); MSL, membranous septum length; RBBB, right bundle branch block; LVOT-EI, eccentricity index of the left ventricular outflow tract; LCC, left coronary cusp; AU, Agatston units. 
available self-expandable device revealed the following findings:

* The need for PPM implantation due to highergrade AVBs was high with 23.1\%;

- Previous RBBB and a pronounced LVOT-EI were independent predictors of PPM need;

* Most of the previously reported determinants failed to predict PPM implantation in patients with STHV-34 including MSL and implantation depth.

Although new generation TAVR devices have improved technologically, a significant (further) reduction in post-procedural cardiac conduction disorders remains desirable for most devices currently available. Intra- and atrioventricular conduction disorders are the most frequent adverse events following TAVR and depend on device type, whereas new LBBB is the most frequent of all conduction abnormalities. Interestingly, in this study, no patient developing post-procedural PPM dependency had previous LBBB or gained LBBB post-procedurally. Furthermore, neither preexisting atrioventricular conduction disorders nor $\mathrm{AF}$ were predictive. In contrast to this, $\mathrm{RBBB}$ alone or in combination with other conduction disturbances was highly predictive for PPM need by univariate and multivariate analysis, being in line with the current research knowledge (12). The left bundle branch emerges from beneath the membranous septum and is located within the interventricular septum. That's why LBBB is the most common conduction disturbance following TAVR, when the native valve is crushed, and the surrounding tissues are stressed by the expanding valve prosthesis. Therefore, procedure-related provocation of new-onset LBBB or high-degree AVB is common, whereas pre-existing LBBB is not handled as strong risk factor for higher-grade conduction disturbances following TAVR. In our study, most of the patients with different pre-existing conduction disturbances switched immediately into a high-degree AVB, so a new-onset of LBBB was not recorded (only as LBBB configuration under escape rhythm/high-degree AVB).

Further identified risk factors for new PPM implantation in self-expandable devices include lower valve implantation towards the left ventricle, a short MSL or implantation depth over MSL, the ratio of bioprosthesis diameter to LVOT diameter and calcium distribution $(12,17,18)$. In our study, there was no association between new PPM and the degree of oversizing or association regarding bioprosthesis to LVOT diameter or aortic root entrance angle. Furthermore, there was no association of new PPM and prosthesis implantation depth towards both leaflets (NCC/
LCC) as well as to MSL or their ratios, being contrary to previous trials. Only implantation depth over MSL revealed a medium correlation for PPM need but failed to be a dependent or independent predictor by further analysis. With a former recommendation of 3-6 $\mathrm{mm}$ of target implantation depth, both centres involved in this study had achieved well implantation results with a mean of $5-7 \mathrm{~mm}$ in projection on the NCC. The membranous septum is often larger/longer than usual (mostly between $0-5 \mathrm{~mm}$ in smaller roots) according to the large aortic root anatomy. Nonetheless, we detected no relationship in cases were the ID exceeded the MSL. Instead, a pronounced eccentricity of the LVOT was an independent predictor for PPM need, arguing for possible shear stress when implanting the device in oval or tubular structures with a smaller minimal diameter as compared to the annulus size.

Aortic valve calcification is strongly required for device anchoring, but extensive calcium deposits may also lead to worse adverse events (coronary obstruction, annular/ventricular perforation, relevant paravalvular regurgitation). However, the association between classical PPM determinants and multi-locular calcium distribution of the aortic root has not been studied with the largest STHV-34 device in detail. Concordant to its smaller siblings, this supra-annular prosthesis is also characterized by a new design with an extended sealing skirt and a more consistent radial force to reduce paravalvular leakage. The annulus coverage is broader regarding annulus range and contact zone, possibly resulting in more stress of nearby structures and challenging PPM predictors. LCC leaflet and DLZ calcification was previously analyzed in particular $(16,18,19)$ and defined as PPM predictor. Additionally, in asymmetrically calcified leaflets, the "contre-coup" phenomenon during valve-deployment was already described (20), leading to a calcification-related stentframe movement towards the opposite side. Furthermore, calcium-free zones favor more direct pressure on the atrioventricular node, possibly being of greater relevance in large self-expandable valves. In our study, in-depth leaflet and ventricular calcification analysis revealed that the amount of LCC calcification was inversely correlated to PPM implantation by univariate analysis. It is well-known that patients who underwent new PPM had higher NCC leaflet calcification in the DLZ or LVOT regions, possibly compressing the cardiac conduction system to a greater extent. On the other side, neither high NCC and or right coronary cusp (RCC) calcification, LVOT calcification beneath the NCC or RCC or MSL-located calcification was 
found to be dependently or independently associated with permanent AVB and PPM in this study.

In this context-regarding the STHV-34 broad annulus coverage and contact area-we postulated that only calcium burden in context with post-dilatation or resheathing maneuvers might be valid predictors in this large device, raising stress on the annular and ventricular circumference. The inconsistent data on STHV-34 PPM incidence ranging from $5-30 \%$ can possibly also be explained by this effect and differing numbers of resheathing or post-dilatation processes. However, in this study, no impact of postdilatation or resheathing maneuvers was observed in the overall PPM-cohort. Remarkably, a high number of patients developed a higher-grade AVB without previous conduction disorders. A sub-analysis of twelve patients with previous SR revealed more impact of calcification distribution in terms of less LCC calcification burden and also impact of pre-dilatation processes, arguing for different patterns of PPM dependency that have to be distinguished in further trials.

\section{Limitations}

The sub-analysis of patients with previous SR developing higher-grade AVBs is obviously underpowered and has to be interpreted with caution, considered that we did not perform separate electrophysiological studies (e.g., HVintervall). However, while implantation of the STHV34 is generally rare as compared to other device-sizes, we offer one of the largest cohorts. The reconstructions were established in diastolic datasets with possible influence on the annulus/LVOT shape. However, the diastolic movement/reconstruction affects all measurements. Regardless of that, we see a strong relationship between a pronounced EI and conduction disturbances leading to PPM.

\section{Conclusions}

This study offers new insights into potential PPM predictors using the STHV-34: previous RBBB and a pronounced LVOT-EI were independent predictors of PPM, while most of the previously reported determinants failed to predict PPM-need including MSL and ID.

\section{Acknowledgments}

We thank Jenni Scharlau for substantial contribution in data acquisition.

Funding: None.

\section{Footnote}

Reporting Checklist: The authors have completed the MDAR reporting checklist. Available at http://dx.doi.org/10.21037/ cdt-20-680

Data Sharing Statement: Available at http://dx.doi. org/10.21037/cdt-20-680

Conflicts of Interest: All authors have completed the ICMJE uniform disclosure form (available at http://dx.doi. org/10.21037/cdt-20-680). Dr. VV, DF, CJ and TZ reports grants, personal fees, non-financial support and other from EDWARDS, grants, personal fees, nonfinancial support and other from MEDTRONIC, outside the submitted work. The other authors have no conflicts of interest to declare.

Ethical Statement: The authors are accountable for all aspects of the work in ensuring that questions related to the accuracy or integrity of any part of the work are appropriately investigated and resolved. All patients provided written informed consent for TAVR and the use of clinical, procedural, and follow-up data for research. The study procedures were in accordance with the Declaration of Helsinki (as revised in 2013). The institutional Ethics Committees of the Heinrich-Heine University (Düsseldorf, 4080) as well as Christian-Albrechts-University (Kiel) approved the study protocol.

Open Access Statement: This is an Open Access article distributed in accordance with the Creative Commons Attribution-NonCommercial-NoDerivs 4.0 International License (CC BY-NC-ND 4.0), which permits the noncommercial replication and distribution of the article with the strict proviso that no changes or edits are made and the original work is properly cited (including links to both the formal publication through the relevant DOI and the license). See: https://creativecommons.org/licenses/by-nc-nd/4.0/.

\section{References}

1. Nkomo VT, Gardin JM, Skelton TN, et al. Burden of valvular heart diseases: a population-based study. Lancet 2006;368:1005-11.

2. Falk V, Baumgartner H, Bax JJ, et al. 2017 ESC/EACTS 
Guidelines for the management of valvular heart disease.

Eur J Cardiothorac Surg 2017;52:616-64.

3. Siontis GCM, Overtchouk P, Cahill TJ, et al.

Transcatheter aortic valve implantation vs. surgical aortic valve replacement for treatment of symptomatic severe aortic stenosis: an updated meta-analysis. Eur Heart J 2019;40:3143-53.

4. Grube E, Van Mieghem NM, Bleiziffer S, et al. Clinical outcomes with a repositionable self-expanding transcatheter aortic valve prosthesis: the international FORWARD study. J Am Coll Cardiol 2017;70:845-53.

5. Kuhn C, Frerker C, Meyer AK, et al. Transcatheter aortic valve implantation with the $34 \mathrm{~mm}$ self-expanding CoreValve Evolut R: initial experience in 101 patients from a multicentre registry. EuroIntervention 2018;14:e301-5.

6. Harnath A, Gomes B, Herwig V, et al. First experience with the $34 \mathrm{~mm}$ self-expanding Evolut $\mathrm{R}$ in a multicentre registry. EuroIntervention 2018;14:e298-300.

7. Eitan A, Witt J, Stripling J, et al. Performance of the Evolut-R 34 mm versus Sapien-3 $29 \mathrm{~mm}$ in transcatheter aortic valve replacement patients with larger annuli: Early outcome results of Evolut-R $34 \mathrm{~mm}$ as compared with Sapien-3 $29 \mathrm{~mm}$ in patients with Annuli $\geq 26 \mathrm{~mm}$. Catheter Cardiovasc Interv 2018;92:1374-9.

8. Khawaja MZ, Rajani R, Cook A, et al. Permanent pacemaker insertion after CoreValve transcatheter aortic valve implantation: incidence and contributing factors (the UK CoreValve Collaborative). Circulation 2011;123:951-60.

9. Jilaihawi H, Chakravarty T, Weiss RE, et al. Meta-analysis of complications in aortic valve replacement: comparison of Medtronic-Corevalve, Edwards-Sapien and surgical aortic valve replacement in 8,536 patients. Catheter Cardiovasc Interv 2012;80:128-38.

10. Hellhammer K, Piayda K, Afzal S, et al. The Latest Evolution of the Medtronic CoreValve System in the Era of Transcatheter Aortic Valve Replacement: Matched Comparison of the Evolut PRO and Evolut R. JACC Cardiovasc Interv 2018;11:2314-22.

11. Manoharan G, Van Mieghem NM, Windecker S, et al. 1-year outcomes with the Evolut R self-expanding transcatheter aortic valve: from the International FORWARD Study. JACC Cardiovasc Interv 2018;11:2326-34.

12. Jilaihawi $H$, Zhao Z, Du R, et al. Minimizing permanent pacemaker following repositionable self-expanding transcatheter aortic valve replacement. JACC Cardiovasc Interv 2019;12:1796-807.

13. Achenbach S, Delgado V, Hausleiter J, et al. SCCT expert consensus document on computed tomography imaging before transcatheter aortic valve implantation (TAVI)/transcatheter aortic valve replacement (TAVR). J Cardiovasc Comput Tomogr 2012;6:366-80.

14. Abramowitz Y, Maeno Y, Chakravarty T, et al. Aortic angulation attenuates procedural success following selfexpandable but not balloon-expandable TAVR. JACC Cardiovasc Imaging 2016;9:964-72.

15. Alqahtani AM, Boczar KE, Kansal V, et al. Quantifying aortic valve calcification using coronary computed tomography angiography. J Cardiovasc Comput Tomogr 2017;11:99-104.

16. Seiffert M, Fujita B, Avanesov M, et al. Device landing zone calcification and its impact on residual regurgitation after transcatheter aortic valve implantation with different devices. Eur Heart J Cardiovasc Imaging 2016;17:576-84.

17. Jilaihawi H, Makkar RR, Kashif M, et al. A revised methodology for aortic-valvar complex calcium quantification for transcatheter aortic valve implantation. Eur Heart J Cardiovasc Imaging 2014;15:1324-32.

18. Fujita B, Kütting M, Seiffert M, et al. Calcium distribution patterns of the aortic valve as a risk factor for the need of permanent pacemaker implantation after transcatheter aortic valve implantation. Eur Heart J Cardiovasc Imaging 2016;17:1385-93.

19. Pollari F, Großmann I, Vogt F, et al. Risk factors for atrioventricular block after transcatheter aortic valve implantation: a single-centre analysis including assessment of aortic calcifications and follow-up. Europace 2019;21:787-95.

20. Maeno Y, Abramowitz Y, Kawamori H, et al. A highly predictive risk model for pacemaker implantation after TAVR. JACC Cardiovasc Imaging 2017;10:1139-47.

Cite this article as: Veulemans V, Frank D, Seoudy H, Wundram S, Piayda K, Maier O, Jung C, Polzin A, Frey N, Kelm M, Zeus T. New insights on potential permanent pacemaker predictors in TAVR using the largest self-expandable device. Cardiovasc Diagn Ther 2020;10(6):1816-1826. doi: $10.21037 / \mathrm{cdt}-20-680$ 

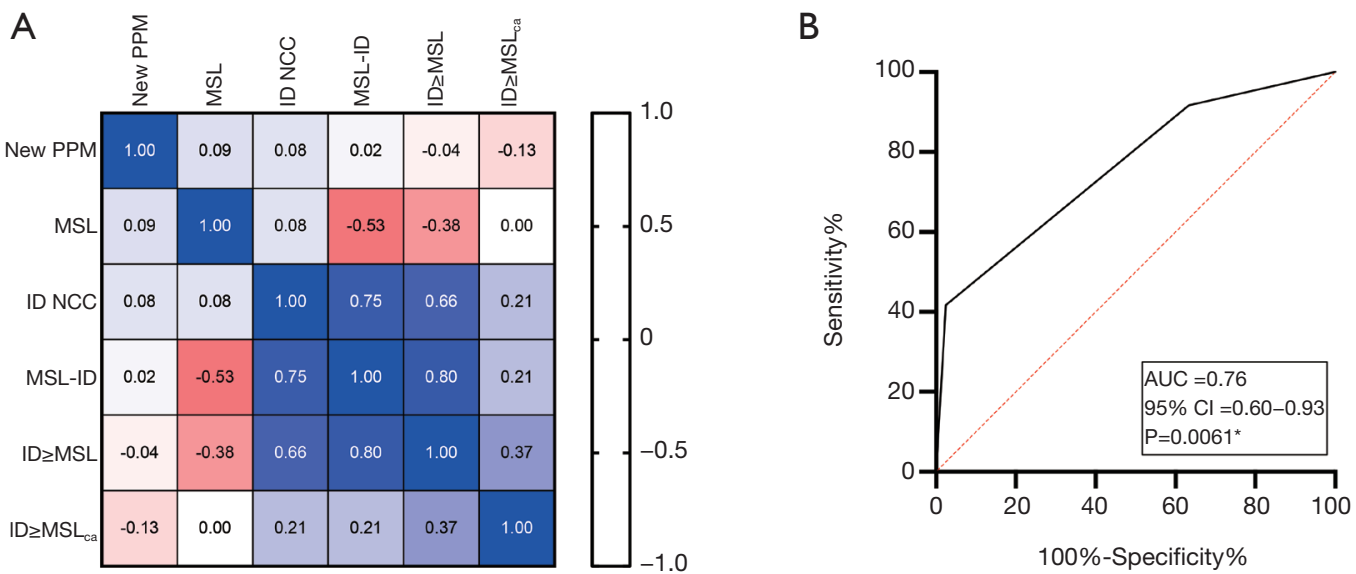

Figure S1 Association of need for PPM with potential and determined predictive factors in patients with SR. (A) Correlation matrix of ID towards NCC, MSL, MSL to ID distance, ID $\geq$ MSL, and ID $\geq M_{\mathrm{Ca}}$. (B) Combined c-statistics of the independent predictors for PPM need (pre-dilatation and LCC calcium burden <762 AU). *, significant $\mathrm{P}$ value $<0.05$. PPM, permanent pacemaker; SR, sinus rhythm; ID, implantation depth; NCC, noncoronary cusp; MSL, membranous septum length; LCC, left coronary cusp; AU, Agatston Units; AUC, area under the curve; CI, confidence interval.

Table S1 Univariate and binary multivariate logistic regression analysis of predictors for PPM in previous SR

\begin{tabular}{|c|c|c|c|c|}
\hline Predictor & \multicolumn{2}{|c|}{ Univariate analysis } & \multicolumn{2}{|c|}{ Multivariate analysis } \\
\hline $\mathrm{BMI} \geq 26$ & $3.83(0.90-16.26)$ & 0.068 & - & - \\
\hline Pre-dilatation & $3.86(0.99-15.07)$ & 0.052 & $8.53(1.23-59.41)$ & $0.030^{\star}$ \\
\hline Post-dilatation & $3.60(0.79-16.49)$ & 0.099 & - & - \\
\hline DLZ-LCC <457 AU & $3.60(0.79-16.49)$ & 0.099 & - & - \\
\hline
\end{tabular}

*, significant $\mathrm{P}$ value $<0.05$. PPM, permanent pacemaker; SR, sinus rhythm; Cl, confidence interval; BMI, body mass index; LCC, left coronary cusp; AU, Agatston Units; DLZ, device landing zone. 